\title{
Comparison of the gut microbiome in red deer (Cervus elaphus) and fallow deer (Dama dama) by high-throughput sequencing of the V3-V4 region of the 16S rRNA gene
}

Xibao Wang $^{\mathrm{a}, \uparrow}$, Yao Chen ${ }^{\mathrm{a}, \uparrow}$, Yongquan Shang ${ }^{\mathrm{a}}$, Xiaoyang $\mathrm{Wu}^{\mathrm{a}}$, Qinguo Wei ${ }^{\mathrm{a}}$, Jun Chen ${ }^{\mathrm{b}}$, Jiakuo Yan $^{\mathrm{a}}$, Honghai Zhang ${ }^{\mathrm{a}, *}$

a College of Life Science, Qufu Normal University, Qufu, Shandong, China

b College of Marine Life Sciences, Ocean University of China, Qingdao, China

$\dagger$ Shared first authorship

*Corresponding author, e-mail: zhanghonghai67@126.com

Received 11 Jun 2019

Accepted 10 Oct 2019

\begin{abstract}
The gut microbiome is a complex ecosystem that plays an essential role in maintaining the host's gut homeostasis and health. The available sequencing data for the Cervidae gut microbiome, especially for red deer and fallow deer in China, are limited. To improve the health of the two species in captivity, we characterized the gut microbiome of red deer and fallow deer via high-throughput Illumina sequencing of the 16S rRNA genes V3-V4 hypervariable regions. We found that the gut microbiome community of red deer included 20 phyla, 89 families and 193 genera, while the gut microbiome community of fallow deer included 18 phyla, 102 families and 227 genera. Firmicutes (red deer: 37.46-44.98\%; fallow deer: 32.67-49.54\%) was the most abundant phylum, followed by Bacteroidetes (red deer: 28.09-39.02\%; fallow deer: 27.45-38.71\%). The observed species and Shannon indices in red deer were higher than in fallow deer ( $p=0.002,0.016$, respectively). Hence compared with fallow deer, red deer had higher diversity and species richness in their gut microbiome. The MRPP results showed that significant differences did occur in their microbiome compositions $(A=0.037$, significance level $\alpha=0.001)$. This study provides an initial understanding of the gut microbiome composition of red deer and fallow deer. The results may benefit captive management and future reintroduction programs.
\end{abstract}

KEYWORDS: red deer (Cervus elaphus), fallow deer (Dama dama), gut microbiome, 16sRNA, high-throughput Illumina sequencing

\section{INTRODUCTION}

The gut microbial community is the key to processing the diet, thus affecting energy harvest and storage $^{1}$. Hence the gut microbiome plays an important role in maintaining the host's health ${ }^{2}$. Many researchers have studied on the gut microbiome of herbivores $^{3-7}$. For example, Firmicutes (68\%), Bacteroidetes (14\%), and Proteobacteria (10\%) predominated among gut bacterial composition of healthy horses ${ }^{5}$. Li et $\mathrm{al}^{8}$ found that Firmicutes (64\%) and Bacteroidetes (22\%) were dominant in the gut microbiome of donkeys, followed by Verrucomicrobia (5\%), Euryarchaeota (4\%), Proteobacteria (3\%), and Spirochaete (2\%). Firmicutes (66.3769.23\%) and Bacteroidetes (21.26-26.55\%) were the major phyla in the gut flora of golden takins with more than $90 \%$ of the total sequences in all samples ${ }^{4}$. Considerable evidence supports the necessity of the gut microbiome for maintaining gut homeostasis in herbivores. For example, compared with healthy musk deer, Escherichia-Shigella and $\mathrm{Fu}$ sobacterium were major bacterial pathogens in the gut microbiota of musk deer with diarrhoea ${ }^{9}$. Costa et $\mathrm{al}^{5}$ found that Actinobacteria and Spirochaetes were major taxa in gut microbiota of healthy horses, while the horses with colitis had a significantly greater abundance of Fusobacteria. If the homeostasis of gut microbiota is disrupted, the host may become ill ${ }^{10}$.

Red deer (Cervus elaphus) also known as wapiti and elk, belong to the deer family Cervidae. Red deer are classified into eight subspecies according to distinct differences in physical characteristics and geographic location. Red deer are under state pro- 
tection (category II) in China. Red deer generally eat high-energy forbs in summer and the standing crops of graminoids in winter ${ }^{11}$ and always lick salt to avoid the low sodium ${ }^{12}$. Fallow deer (Dama dama) also belong to the deer family Cervidae and are native to Europe. Fallow deer has been introduced in many countries and areas, including China, South Africa, Peru, and Israel. The species Dama comprises two subspecies, the European fallow deer (Duma duma duma) and the Persian or Mesopotamian fallow deer (Duma duma mesopofamica $^{13,14}$. Fallow deer prefer to eat all types of common sub canopy trees, including broadleaf ${ }^{15}$. Fallow deer also eat sedges, rushes and heather, which account for approximately $30 \%$ of the diet ${ }^{16}$.

The two Cervidae species have economic and medicinal value in the velvet antlers, meat and skin. Red deer and fallow deer have a long history of feeding in Xinjiang, China, and Europe ${ }^{17}$. At present, partial research has been done for red deer and fallow deer on the basis of molecular biology ${ }^{18,19}$. Most of this has research focused on population, biology and trophic analyses ${ }^{20-22}$. While the rumen microbiome of red deer in a captive environment and the impact of winter enclosures on the gut microbiome of red deer have been studied previously ${ }^{23,24}$, the gut microbiomes of red deer and fallow deer in captive environments have not previously been researched. Hence we characterized the gut microbiome composition of fallow deer and red deer by high-throughput sequencing. We also compared gut microbiome diversity of fallow deer and red deer living in the same captive environment. The results may contribute to improving the health of captive red deer and fallow deer and may benefit captive management and future reintroduction programs.

\section{MATERIALS AND METHODS}

\section{Sample collection}

Sixteen fresh fecal samples of fallow deer (group TL) and eight fresh fecal samples of red deer (group ML) were collected in Shijiazhuang Zoo in December, 2017. The red deer and fallow deer were living in the same captive environment; under the same temperature $\left(-10^{\circ} \mathrm{C}\right.$ to $\left.-15^{\circ} \mathrm{C}\right)$ and the same captive management method. The fallow deer and red deer food was a mixed fodder based on corn stalks. Protocols used for this experiment were consistent with those approved by the Institutional Animal Care and Committee at Qufu Normal University. All animals were healthy adults and given no medica- tion within the prior four months before sampling. Before feeding in the morning, fresh fecal samples were immediately collected after defecation and put into sterile plastic tubes. The samples were carried to the laboratory and stored at $-80^{\circ} \mathrm{C}$.

\section{DNA extraction, 16S rRNA gene amplification by PCR and sequencing}

(1) Total genomic DNA was extracted using a QIAamp Stool Mini KIT (Qiagen, Germany) following the manufacturer's recommendations. In our experiment, the concentration of DNA was measured by Qubit 2.0 to ensure that the concentration was higher than $20 \mathrm{ng} / \mu \mathrm{l}$.

(2) The V3-V4 hypervariable region of the bacterial 16S rRNA gene was amplified using universal amplicon PCR primers: forward primer (CTACG GGNGGCWGCAG) and reverse primer (GACTA CHVGGGTATCTAATCC). The total final mixture for the polymerase chain reaction volume was $50 \mu \mathrm{l}: 5 \mu \mathrm{l}$ of microbial DNA ( $5 \mathrm{ng} / \mu \mathrm{l}), 25 \mu \mathrm{l}$ of $2 \times$ Taq PCR Master Mix (0.1 U/ $\mu 1) ; 18 \mu l$ of $\mathrm{ddH}_{2} \mathrm{O}$ and $2 \mu \mathrm{l}$ of each primer $(10 \mu \mathrm{M})$.

(3) The PCR amplification conditions were as follows: $1 \mathrm{~min}$ at $98^{\circ} \mathrm{C}$ for pre-denaturation and then 25 cycles of $30 \mathrm{~s}$ at $95^{\circ} \mathrm{C}$ for denaturation, 30 seconds at $55^{\circ} \mathrm{C}$ for annealing and $30 \mathrm{~s}$ at $72{ }^{\circ} \mathrm{C}$ for elongation, followed by $5 \mathrm{~min}$ at $72{ }^{\circ} \mathrm{C}$ for the final extension.

(4) The PCR products were mixed with $1 \times$ loading buffer (containing SYBR green) and checked on $2 \%(\mathrm{w} / \mathrm{v})$ agarose gels in TBE buffer (Tris, boric acid, EDTA) by electrophoresis. After the gel electrophoresis, the PCR product was examined for the presence of bright band between 400 and 450 bp under a UV lamp. Finally, the DNA target strip was purified with a Qiagen Gel Extraction Kit (Qiagen, Germany).

(5) The sequencing libraries were generated using a TruSeq PCR-Free DNA Sample Preparation Kit (Illumina, USA); following the manufacturer's recommendations and index codes were added $^{9}$. The sequencing library quality was evaluated via Qubit 2.0 Fluorometer (Thermo Scientific) and an Agilent Bioanalyzer 2100 system. Finally, the DNA sequencing libraries were sequenced on the Illumina MiSeq system (Illumina MiSeq, USA), following the manufacturer's instructions.

\section{Sequence processing and analysis}

(1) Paired-end reads were assigned to samples based on the unique barcode and were trun- 
cated by cutting the barcode and primer sequences. Paired-end reads were combined by using FLASH (V1.2.7) ${ }^{25}$.

(2) The raw tags were used to perform specific filtering to obtain high-quality clean tags by using QIIME (V1.9.1) ${ }^{26}$. The tags were compared with the database (Gold database) using the UCHIME algorithm to remove the chimera sequences in order to obtain the final effective $\operatorname{tags}^{26}$.

(3) The effective tags clustered at greater than or equal to $97 \%$ similarity were distributed to the same operational taxonomic unit (OTU) by UPARSE software $(V 7.0 .1001)^{27}$. Species annotations were based on the RDP classifier algorithm ${ }^{28}$ using the GreenGenes database ${ }^{29}$.

(4) Alpha diversity and Beta diversity were calculated with QIIME (V1.9.1) ${ }^{26}$, including the observed-species, Chao1, Shannon, Simpson, ACE, and Good-coverage indices. The Rarefaction Curves, Rank Abundance Curves, and principal component analysis (PCA) were drawn using the FactoMineR and ggplot2 packages in R software (V2.15.3).

(5) We chose multi-response permutation procedures (MRPP) analysis to test the differences between groups. MRPP analysis was done with the 'mrpp' function of the $\mathrm{R}$ vegan package.

The data set supporting the results of this article can be found in the Sequence Read Archive database, accession number SUB5086400.

\section{RESULTS}

A total of 2749256 quality reads (734601 for red deer and 2014655 for fallow deer) were obtained after quality control from the 24 samples. The quality reads were classified into 2092 OTUs (red deer) and 2527 OTUs (fallow deer) using the $97 \%$ similarity criterion. The high-quality reads had an average length of $410 \mathrm{bp}$. Based on a genetic distance of $3 \%$, we observed a significant difference $(p<0.05)$ between group ML and group TL by using MRPP analysis. The alpha index is listed in Table 1 (including observed species, Shannon, Simpson, Chao1, ACE, and Good coverage). The rarefaction curves became gradually wider and approached with more data indicating that the samples sizes from the sequencing were reasonable (Fig. 1A). The rank abundance curves reflect the evenness and abundance of species in the fecal samples both horizontally and vertically (Fig. 1B).

\section{Bacterial composition and relative abundance}

At the phylum level, we observed 20 phyla and 18 phyla from 8 red deer samples and 16 fallow deer samples, respectively. We used the largest abundance of the top 10 phyla to generate a relative abundance column cumulative plot (Fig. 2A). Firmicutes (group ML: 41.86 $\pm 2.27 \%$; group TL: $42.01 \pm 4.15 \%$ ) and Bacteroidetes (group ML: $32.86 \pm 3.94 \%$; group TL: $32.72 \pm 3.22 \%$ ) dominated the gut microbiota of red deer and fallow deer (Fig. 2A). The abundance of the two phyla was more than $70 \%$ of the total reads in 24 samples. In addition, other major phyla were Spirochaetes (group ML: $4.54 \pm 2.03 \%$; group TL: $5.17 \pm 3.10 \%$ ), Proteobacteria (group ML: $4.85 \pm 1.85 \%$; group TL: $6.30 \pm 2.60 \%$ ), and Euryarchaeota (group ML: $4.48 \pm 1.98 \%$; group TL: $3.31 \pm 2.30 \%$ ). There were unclassified bacteria in the gut microbiota of fallow deer $(0.32 \%)$ and red deer $(0.25 \%)$, indicating some novel bacteria existed.

At the family level, we observed 89 families and 102 families from 8 red deer samples and 16 deer samples, respectively. We used the largest abundance of the top 10 families to generate a relative abundance column cumulative plot (Fig. 2B). These families occupied more than $68 \%$ of the gut microbiota of the two species. Among these 10 families, Ruminococcaceae (group ML: $25.66 \pm 1.23 \%$; group TL: $24.47 \pm 3.28 \%$ ) was the largest in abundance, while Bacteroidales RF16 group was the least abundant bacterial (group ML: $2.22 \pm 1.32 \%$; group TL: $2.23 \pm 1.99 \%$ ). We compared the difference of gut bacteria composition between red deer and fallow deer.

At the genus level, we observed 193 genera and 227 genera from 8 red deer samples and 16 fallow deer samples, respectively. We used the largest abundance of the top 10 genera to generate relative abundance column cumulative plot (Fig. 2C). The abundance of the top 10 bacteria genera comprised more than $43 \%$ of the gut microbiota of the two species. Among these 10 genera, $R u$ minococcaceae UCG-010 (group ML: 10.98 $\pm 1.52 \%$; group TL: $10.42 \pm 1.93 \%$ ) was the largest in abundance, while Methanocorpusculum was the least abundant bacterial (group ML: $1.09 \pm 1.29 \%$; group TL: $0.66 \pm 1.47 \%$ ). The top 10 bacteria genera were not significantly different between fallow deer and red deer. This reflected the basic structure of the gut microbiota composition in red deer and fallow deer. Combined with the above results, this indicated that major bacteria were dominant in the 
Table 1 Alpha-diversity of the gut microbiota in fecal samples from the red deer (group ML) and fallow deer (group TL).

\begin{tabular}{lrlrrrr}
\hline Sample & $\begin{array}{c}\text { Observed } \\
\text { species }\end{array}$ & Shannon & Simpson & Chao1 & ACE & $\begin{array}{c}\text { Goods } \\
\text { coverage }\end{array}$ \\
\hline ML1 & 1216 & 8.367 & 0.991 & 1294.50 & 1309.73 & 0.995 \\
ML2 & 1414 & 8.755 & 0.995 & 1618.40 & 1606.08 & 0.992 \\
ML3 & 1359 & 8.097 & 0.988 & 1644.24 & 1613.38 & 0.991 \\
ML4 & 1359 & 8.687 & 0.994 & 1439.96 & 1469.71 & 0.995 \\
ML5 & 1166 & 8.11 & 0.99 & 1373.14 & 1317.96 & 0.994 \\
ML6 & 1365 & 8.207 & 0.99 & 1606.97 & 1601.54 & 0.991 \\
ML7 & 1424 & 8.67 & 0.994 & 1670.34 & 1618.76 & 0.992 \\
ML8 & 1447 & 8.729 & 0.994 & 1672.93 & 1637.91 & 0.992 \\
TL1 & 1160 & 8.316 & 0.993 & 1322.43 & 1327.13 & 0.994 \\
TL2 & 1258 & 8.095 & 0.989 & 1508.45 & 1465.70 & 0.992 \\
TL3 & 1402 & 8.221 & 0.988 & 1709.71 & 1672.76 & 0.991 \\
TL4 & 938 & 7.583 & 0.987 & 115.00 & 1120.78 & 0.994 \\
TL5 & 1375 & 8.472 & 0.993 & 1590.27 & 1598.89 & 0.992 \\
TL6 & 1237 & 8.482 & 0.993 & 1451.22 & 1425.70 & 0.993 \\
TL7 & 1268 & 8.395 & 0.992 & 1566.41 & 1480.38 & 0.992 \\
TL8 & 1240 & 8.515 & 0.994 & 1416.16 & 1400.50 & 0.994 \\
TL9 & 1299 & 8.387 & 0.993 & 1456.13 & 1473.92 & 0.993 \\
TL10 & 809 & 7.148 & 0.977 & 960.64 & 937.91 & 0.995 \\
TL11 & 846 & 7.166 & 0.977 & 1051.10 & 1044.49 & 0.994 \\
TL12 & 819 & 7.171 & 0.978 & 992.47 & 989.83 & 0.995 \\
TL13 & 881 & 7.323 & 0.98 & 948.50 & 976.86 & 0.996 \\
TL14 & 1355 & 8.759 & 0.995 & 1453.46 & 1461.37 & 0.995 \\
TL15 & 824 & 7.261 & 0.978 & 877.16 & 880.94 & 0.997 \\
TL16 & 1177 & 8.502 & 0.994 & 1271.25 & 1262.76 & 0.995 \\
\hline
\end{tabular}
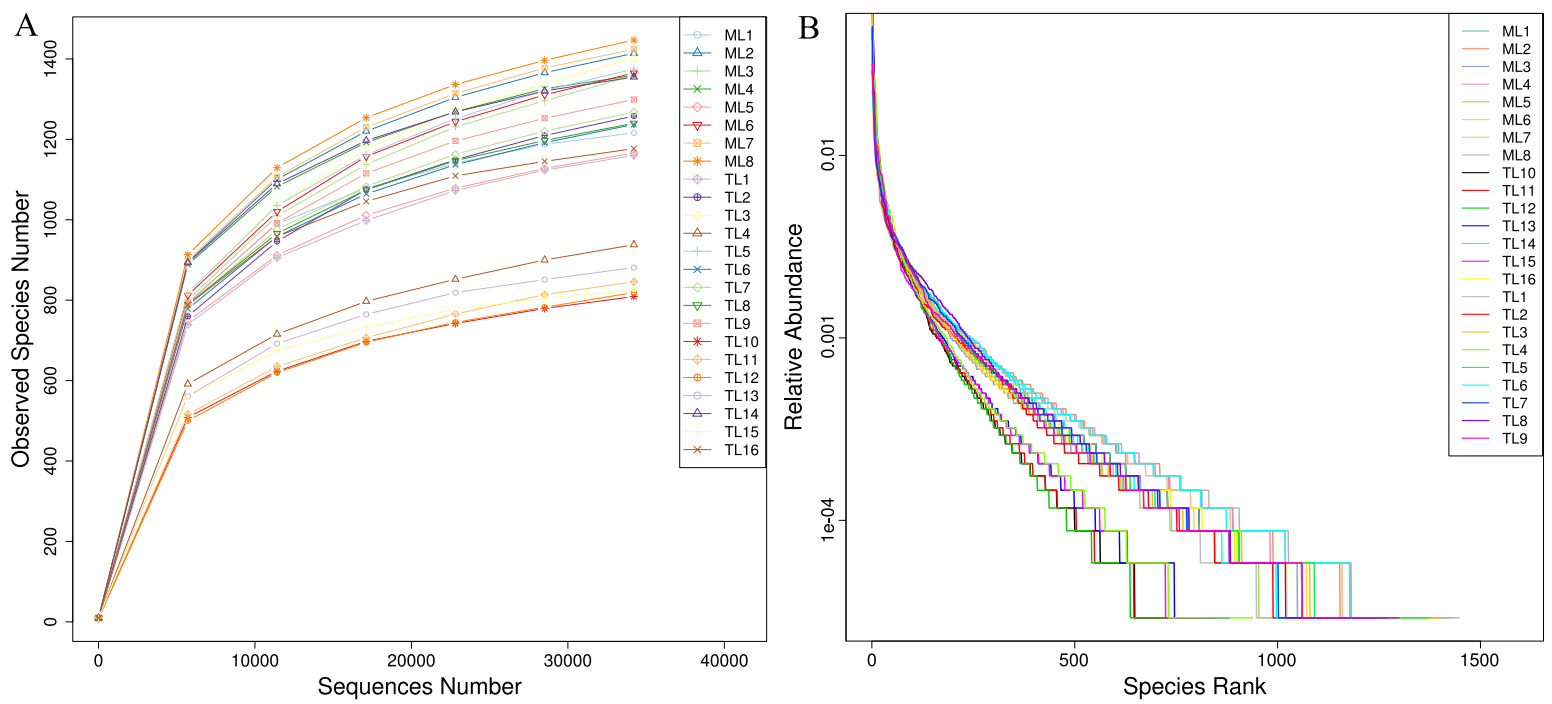

Fig. 1 Rarefaction curves (A) and rank abundance curves (B). In the rarefaction curve, the abscissa is the number of sequencing samples randomly chosen from the sample, and the ordinate is the number of OTUs that can be constructed based on the number of sequencing fragments to reflect the sequencing depth. In the rank abundance curve, the abscissa is the number of OTUs sorted by the abundance of OTUs, and the ordinate is the relative abundance of the corresponding OTUs. 

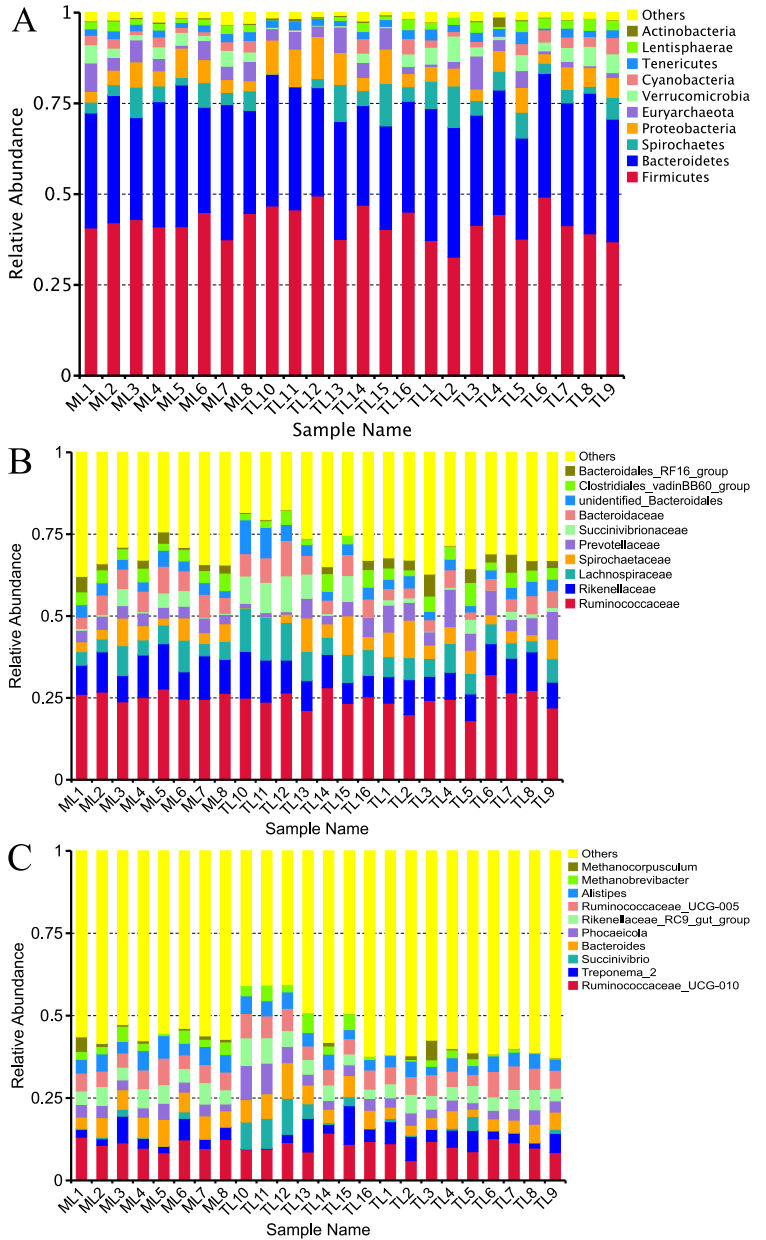

Fig. 2 Fecal microbial composition of red deer and fallow deer at (A) the phylum, (B) family, and (C) genus levels. Each bar represents the top ten bacterial species sorted by relative abundance in each individual sample.

gut microbiota composition and played important role in maintaining the gut microbiota system of herbivores.

\section{Analysis of discrepancies between group}

The observed species and Shannon indices bar plots for red deer and fallow deer are shown in Fig. 3. The gut bacterial diversity (the observed species index between groups: $p=0.002$ ) and richness were significantly different (Shannon index between groups: $p=0.016$ ) between group ML and group TL. We used MRPP to test whether the division of the groups was reasonable $(A=0.037$; significance level $\alpha=$ $0.001)$. The results supported our original designation of the red deer and fallow deer groups. The clustered heatmap (genus level) indicated that red deer were grouped together (group ML: ML1-ML8), and fallow deer were grouped separately (group TL: TL1-TL16). The top 35 genera accounted for the difference in proportions; these are indicated by different colors and locations of clustering in the heatmap (Fig. 4). We plotted a distance matrix from the heatmap by calculating Weighted Unifrac and Unweighted Unifrac distances. By comparing the distances between individuals, the difference between groups was greater than the difference within the group. The results were similar to the clustered heatmap that the difference between groups was greater than within groups (Fig. 5A). From the UPGMA (unweighted Unifrac distance) tree (Fig. 5B), we concluded that fallow deer samples (group TL, excluded TL14) grouped together, while red deer samples (group ML) grouped separately. Furthermore, the separation of group ML from group TL was illustrated by the PCA plot (Fig. 5C).

At each level, significant species-specific differences were indicated in Fig. 6 ( $t$-test). At the order level (Fig. 6A), Planctomycetales was more abundant in group ML than in group TL $(p=0.04)$. The abundance of Anaerolineales was much greater in group TL than in group ML $(p=0.004)$. At the family level (Fig. 6B), Anaerolineaceae was significantly more abundant in group TL than in group ML, and the other three bacteria were significantly more abundant in group ML than in group TL $(p<$ 0.05). At the genus level (Fig. 6C), a total of 13 genera showed significant differences $(p<0.05)$ between group ML and group TL. The abundances of dgA-11 gut group, Ruminococcus 2, CPla-4 termite group and Phascolarctobacterium were higher in red deer than in fallow deer. Other genera were more abundant in the gut microbiota of fallow deer $(p<$ 0.05).

\section{DISCUSSION}

We used high-throughput sequencing to describe the gut microbial community of fallow deer and red deer in the same captive environment. Firmicutes and Bacteroidetes dominated the gut microbiota composition of red deer and fallow deer. The results were similar to those from other studies of herbivores in that Firmicutes and Bacteroidetes were the largest components of the gut microbiome in golden takins ${ }^{4}$, horses ${ }^{5}$, sika deer ${ }^{30}$, and donkeys ${ }^{7}$. Among those results, Costa et $\mathrm{al}^{5}$ and Liu et $\mathrm{al}^{7}$ described the gut bacteria composition of horses and donkeys by V3-V5 and V5-V6 regions of the 16S rRNA gene, respectively. The results were also similar to those from several carnivores; for example, Wu 

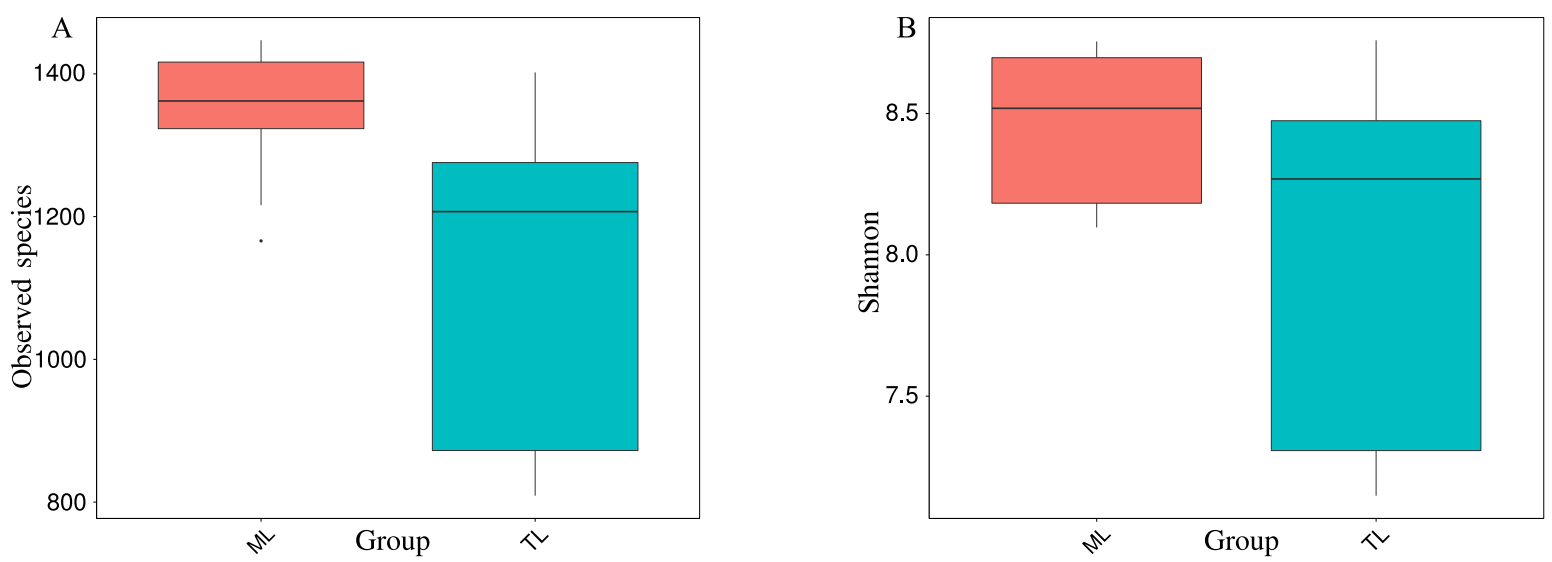

Fig. 3 The comparisons for alpha-diversity between red deer and fallow deer. (A) Richness was determined by calculating the observed-species index, and (B) diversity was measured by calculating the Shannon index.

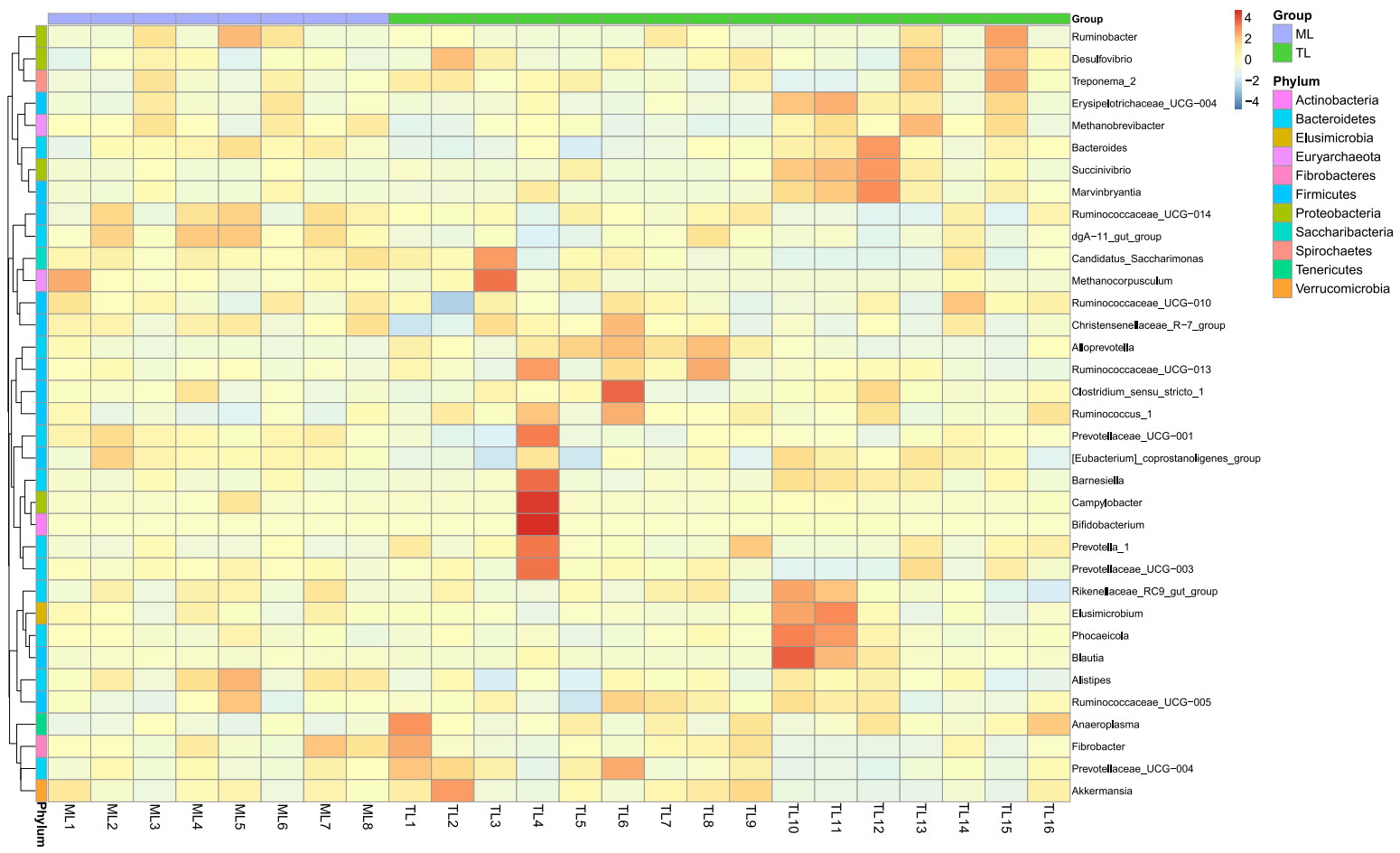

Fig. 4 The heatmap of clustering for species abundance. The abundances of the top 35 genera were sorted for the analysis. The heatmap plot depicts the relative percentage of each bacterial genus (variables clustering on the $y$-axis) within each sample ( $x$-axis clustering).

et $\mathrm{al}^{31}$ discovered that Firmicutes and Bacteroidetes were the most abundant groups in the gut bacterial composition of the dhole.

The bacterial composition was different in the gut and rumen of red deer ${ }^{24}$. Volatile fatty acids (VFAs) formed by the action of Bacteroidetes of rumen microbiome degrading starch, xylan, lignans, and pectin are largely absorbed across the host's ruminal epithelium ${ }^{30}$. The high-fiber diet results in Firmicutes being most abundant in the gut composition of the red deer and fallow deer. Hence Bacteroidetes $(36.2 \pm 3.2 \%)$ was the second most abundant group in the gut microbiome of red deer ${ }^{24}$. In our study, the abundances of Bacteroidetes and Firmicutes were similar to those of captive sika deer. There was a significantly 


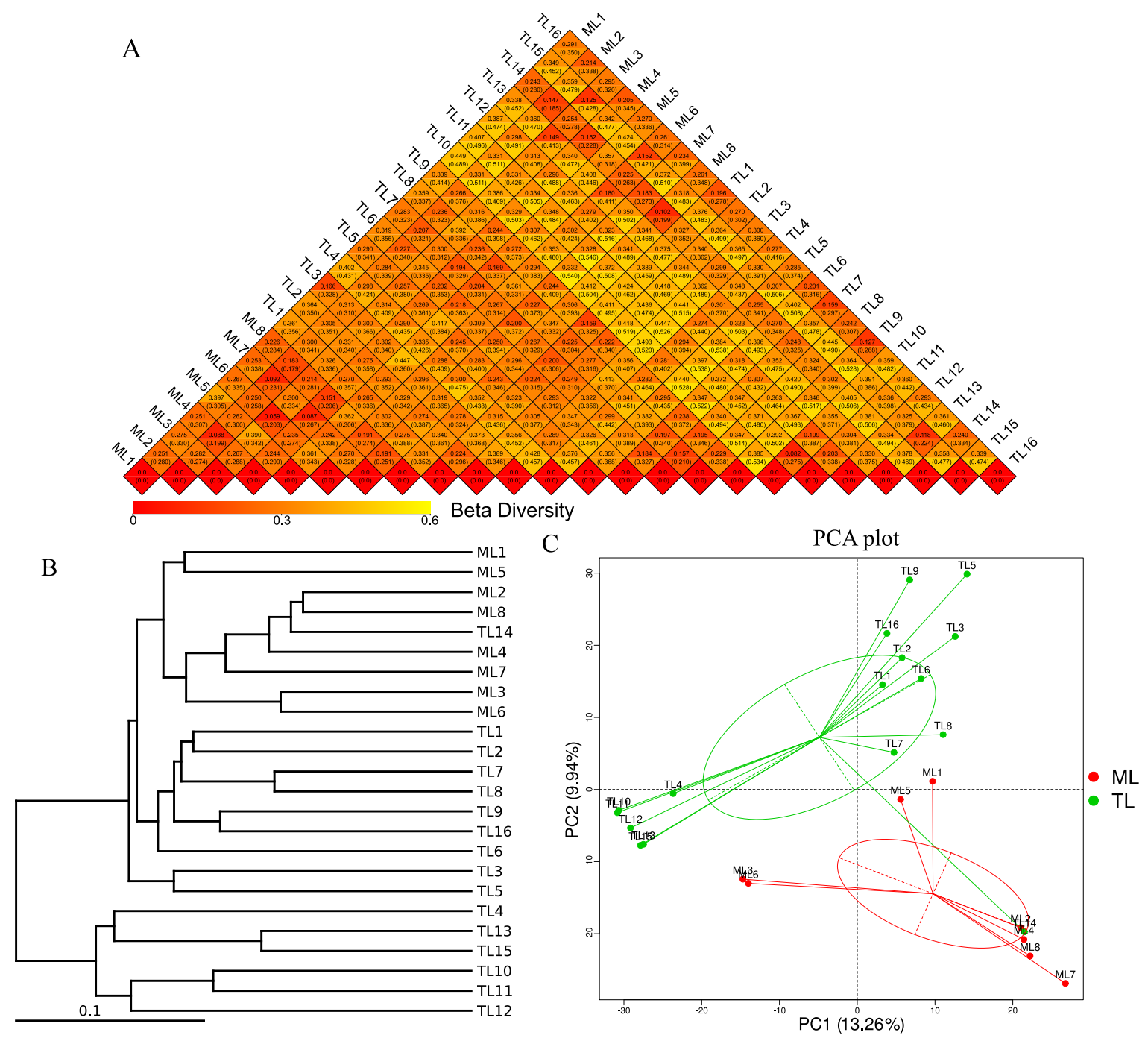

Fig. 5 Heatmap of weighted Unifrac and unweighted Unifrac distances (A) the two numbers in the same grid represent weighted and unweighted Unifrac distances. UPMGA clustering trees (B) based on unweighted Unifrac distance. The results of clustering using distance matrices were combined with the overall percentages of relative abundance among all samples at the phylum level. PCA of bacterial community structures of the gut microbiota in the two sample groups (C) the red and green dots represent red deer and fallow deer samples, respectively.

higher $(p<0.01)$ proportion of Bacteroidetes in captive sika deer $(31.99 \%)$ compared with wild group $(18.28 \%)^{30}$. Our research compared red deer in the enclosures with those in the wild (red deer in enclosures: $75.55 \pm 7.45 \%$; free-ranging red deer: $60.66 \pm 19.24 \%$ ). The result suggests that the abundance of Firmicutes in the gut microbiome of the red deer and fallow deer in a captive environment has been sharply reduced, while there has been a substantial increase in the proportion of Bacteroidetes ${ }^{23}$. The high abundance of Bacteroidetes and lower abundance of Firmicutes in red deer and fallow deer were correlated with dietary proportions in captive deer. The mixed fodder based on corn stalks may be unhealthy for Cervidae.

The rarefaction curves revealed lower phylogenetic diversity and fewer OTUs in the fallow deer microbiome than in the red deer microbiome. The gut microbiome of red deer exhibited significantly higher diversity and richness (Fig. 3). Additionally, the MRPP and Fig. 3 show the separation observed between the gut bacteria composition of group ML and group TL. Lyu ${ }^{32}$ discovered that the different gut compositions and functions of dogs and wolves 


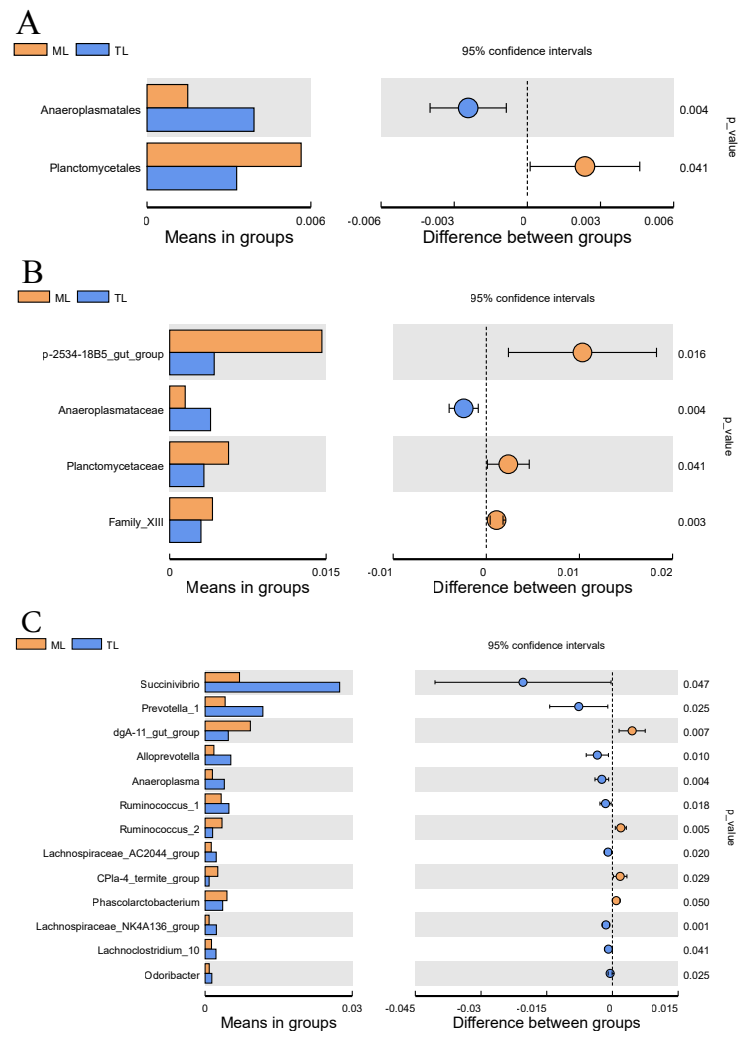

Fig. $6 t$-test difference group analysis diagram: (A) at the order level, (B) at the family level, and (C) at the genus level.

yielded differences in the ability to adapt to a captive environment. The difference in the ability to adapt to a captive environment may result from the different gut bacterial composition of red deer and fallow deer.

At the order level, Planctomycetales belongs to Planctomycetes, and Planctomycetes is often found in the digestive tracts of ruminant animals ${ }^{8}$. This taxon possesses the ability to oxidize ammonium ${ }^{33}$. The phylum Planctomycetales might play an important role in the prevention of disease by reducing the concentration of ammonium in the intestinal system through the ammonium oxide pathway. At the family level, Anaerolineaceae is a family of methanogenic bacteria from the order Anaerolineales $^{34}$. Methanogenic bacteria can convert inorganic or organic compounds into methane by anaerobic fermentation, thus causing energy loss ${ }^{35}$. The abundance of Anaerolineaceae in fallow deer was higher than in red deer, and this may lead to a lower energy yield from the gut microbiome of fallow deer. The abundance of p-2534-18B5 gut group and
Family XIII in red deer was higher than in fallow deer, and Family XIII and p-2534-18B5 gut group belong to Firmicutes and Bacteroidetes, respectively. The difference in bacterial abundance of the two groups may be related to the digestion of crude fiber to produce butyrate ${ }^{5}$.

At the genus level, a total of 13 highly abundant genera were significantly different between fallow deer and red deer. Nine of 13 genera belong to Bacteroidetes and Firmicutes. The significant difference indicated that red deer and fallow deer may have different decomposition abilities for different types of fibers, fats, and proteins. The CPla-4 termite group belongs to Planctomycetes, and the function may be correlated with reducing the concentration of ammonium in the intestinal system ${ }^{8}$. Anaeroplasma has enzymic activity in the Embden-Meyerhof-Parnas pathway for glycolytic catabolism ${ }^{36}$. The high abundance of Anaeroplasma in fallow deer may produce large amounts of lactic acid through anaerobic glycolysis. The high abundance of Succinivibrio has been associated with the high methane yield phenotype ${ }^{37}$. Henderson et $\mathrm{al}^{38}$ reported a positive association between succinate-producing Succinivibrionaceae (Succinivibrio belongs to the family Succinivibrionaceae) and methanogens belonging to the family Methanomassiliicoccaceae. The high abundance of Succinivibrio in the gut microbiome of fallow deer may produce greater amounts of methane, thus removing energy. We speculated that red deer may have a stronger ability to adapt to the captive environment.

In conclusion, we described the dominant gut microbiome populations in red deer and fallow deer. We revealed the diversity of the gut microbiome composition in red deer and fallow deer living in the same captive environment. The characterization of the microbiome composition in red deer and fallow deer has increased our understanding of the bacterial ecosystems in the two species. We speculated that red deer may have a stronger ability to adapt to captive environment. However, further research is needed to confirm this speculation. The results demonstrated that the more detailed information concerning the gut microbiota of red deer and fallow deer in a captive environment can provide us with information useful for improving the health of captive species. In the future, the proportion of feed is an area for improvement according to the focal wildlife animal. The current results provide a basis for captive management and future reintroduction plans. 
Acknowledgements: This work was supported by the Special Fund for Forest Scientific Research in the Public Welfare (201404420) and the National Natural Science Fund of China (31872242, 31672313, 31372220). We thank Shi Jiazhuang Zoo for their assistance in the fecal samples collection. We thank our mentor for their guidance in experiments and writing. We thank LetPub (www.letpub.com) for its linguistic assistance during the preparation of this manuscript. Authors' contributions: conceived and designed the experiments, HHZ, XBW, YC; performed the experiments, XBW, YC, YQS; contributed materials, XBW, JKY; wrote the manuscript, XBW; modified the manuscript, XYW, QGW. All authors read and approved the final manuscript.

\section{REFERENCES}

1. Bäckhed F, Ding H, Wang T, et al (2004) The gut microbiota as an environmental factor that regulates fat storage. Proc Natl Acad Sci USA 101, 15718-15723.

2. Andersson AF, Lindberg M, Jakobsson H, et al (2008) Comparative analysis of human gut microbiota by barcoded pyrosequencing. PloS One 3, ID e2836.

3. Bian G, Ma L, Su Y, et al (2013) The microbial community in the feces of the white rhinoceros (Ceratotherium simum) as determined by barcoded pyrosequencing analysis. PloS One 8, ID e70103.

4. Chen J, Zhang H, Wu X, et al (2017) Characterization of the gut microbiota in the golden takin (Budorcas taxicolor bedfordi). AMB Express 7, ID 81.

5. Costa MC, Arroyo LG, Allen-Vercoe E, et al (2012) Comparison of the fecal microbiota of healthy horses and horses with colitis by high throughput sequencing of the V3-V5 region of the 16S rRNA gene. PloS One 7, ID e41484.

6. Miller H, Carden RF, Evans J, et al (2016) Dead or alive? Investigating long-distance transport of live fallow deer and their body parts in antiquity. Environ Archaeol 21, 246-259.

7. Liu X, Fan H, Ding X, et al (2014) Analysis of the gut microbiota by high-throughput sequencing of the V5-V6 regions of the 16S rRNA gene in donkey. Curr Microbiol 68, 657-662.

8. Li Y, Ma S, Zhang X, et al (2014) Evaluation of bacterial and archaeal diversity in the rumen of Xiangxi yellow cattle (Bos taurus) fed Miscanthus sinensis or common mixed feedstuff. Ann Microbiol 64, 1385-1394.

9. Huptas C, Scherer S, Wenning M (2016) Optimized Illumina PCR-free library preparation for bacterial whole genome sequencing and analysis of factors influencing de novo assembly. BMC Res Notes 9, ID 269.

10. Al Jassim RAM, Andrews FM (2009) The bacterial community of the horse gastrointestinal tract and its relation to fermentative acidosis, laminitis, colic, and stomach ulcers. Vet Clin N Am Equine 25, 199-215.
11. Merrill EH (1994) Summer foraging ecology of wapiti (C. elaphus roosevelti) in the Mount St. Helens blast zone. Can J Zool 72, 303-311.

12. Dalke PD, Beeman RD, Kindel FJ, et al (1965) Use of salt by elk in Idaho. $J$ Wildl Manag 29, 319-332.

13. Chapman NG, Chapman DI (1980) The distribution of fallow deer: a worldwide review. Mammal Rev 10, 61-138.

14. Nam YD, Jung MJ, Roh SW, et al (2011) Comparative analysis of Korean human gut microbiota by barcoded pyrosequencing. PloS One 6, ID e22109.

15. Nugent G (1990) Forage availability and the diet of fallow deer (Dama dama) in the Blue Mountains, Otago. New Zeal J Ecol 13, 83-95.

16. Putman RJ, Culpin S, Thirgood SJ (1993) Dietary differences between male and female fallow deer in sympatry and in allopatry. $J$ Zool 229, 267-275.

17. Ainiwaer $\mathrm{T}$ (2008) The present status and prospects of research on red deer (C. elaphus) three subspecies in XinJiang, China. Xin Jiang Agric Sci 45, 504-551.

18. Bana NÁ, Nyiri A, Nagy J, et al (2018) The red deer $C$. elaphus genome CerEla1. 0: sequencing, annotating, genes, and chromosomes. Mol Genet Genomics 293, 665-684.

19. Meiri M, Kosintsev P, Conroy K, et al (2018) Subspecies dynamics in space and time: a study of the red deer complex using ancient and modern DNA and morphology. J Biogeogr 45, 367-380.

20. Jackowiak H, Skubis J, Łakomy P, et al (2017) Anatomy of the tongue and microstructure of the lingual papillae in the fallow deer Dama dama (Linnaeus, 1758). Mamm Biol 85, 14-23.

21. Hagen R, Haydn A, Suchant R (2018) Estimating red deer (C. elaphus) population size in the Southern Black Forest: the role of hunting in population control. Eur J Wildl Res 64, ID 42.

22. Santos JPV, Vicente J, Carvalho J, et al (2018) Determining changes in the nutritional condition of red deer in Mediterranean ecosystems: Effects of environmental, management and demographic factors. Ecol Indic 87, 261-271.

23. Menke S, Heurich M, Henrich M, et al (2019) Impact of winter enclosures on the gut bacterial microbiota of red deer in the Bavarian Forest National Park. Wildl Biol 2019, ID 503.

24. Qian W, Li ZP, Ao W, et al (2017) Bacterial community composition and fermentation in the rumen of Xinjiang brown cattle (Bos taurus), Tarim red deer (C. elaphus yarkandensis), and Karakul sheep (Ovis aries). Can J Microbiol 63, 375-383.

25. Magoč T, Salzberg SL (2011) FLASH: fast length adjustment of short reads to improve genome assemblies. Bioinformatics 27, 2957-2963.

26. Caporaso JG, Kuczynski J, Stombaugh J, Bittinger K, Bushman FD, Costello EK, Fierer N, Peña AG, et al (2010) QIIME allows analysis of high- 
throughput community sequencing data. Nat Methods 7, 335-336.

27. Edgar RC (2013) UPARSE: highly accurate OTU sequences from microbial amplicon reads. Nat Methods 10, 996-998.

28. Wang Q, Garrity GM, Tiedje JM, et al (2007) Naive Bayesian classifier for rapid assignment of rRNA sequences into the new bacterial taxonomy. Appl Environ Microbiol 73, 5261-5267.

29. DeSantis TZ, Hugenholtz P, Larsen N, et al (2006) Greengenes, a chimera-checked 16S rRNA gene database and workbench compatible with ARB. Appl Environ Microbiol 72, 5069-5072.

30. Guan Y, Yang H, Han S, et al (2017) Comparison of the gut microbiota composition between wild and captive sika deer (Cervus nippon hortulorum) from feces by high-throughput sequencing. AMB Express 7, ID 212.

31. Wu X, Zhang H, Chen J, et al (2016) Comparison of the fecal microbiota of dholes high-throughput Illumina sequencing of the V3-V4 region of the 16S rRNA gene. Appl Microbiol Biotechnol 100, 3577-3586.

32. Lyu T, Liu G, Zhang H, et al (2018) Changes in feeding habits promoted the differentiation of the composition and function of gut microbiotas be- tween domestic dogs (Canis lupus familiaris) and gray wolves (Canis lupus). Amb Express 8, 123.

33. Fuerst JA, Sagulenko E (2011) Beyond the bacterium: planctomycetes challenge our concepts of microbial structure and function. Nat Rev Microbiol 9, 403-413.

34. Liang B, Wang LY, Mbadinga SM, et al (2015) Anaerolineaceae and Methanosaeta turned to be the dominant microorganisms in alkanes-dependent methanogenic culture after long-term of incubation. Amb Express 5, ID 37.

35. Morgavi DP, Forano E, Martin C, et al (2010) Microbial ecosystem and methanogenesis in ruminants. Animal 4, 1024-1036.

36. Petzel JP, Hartman PA (1990) Aromatic amino acid biosynthesis and carbohydrate catabolism in strictly anaerobic mollicutes (Anaeroplasma spp.). Syst Appl Microbiol 13, 240-247.

37. Kamke J, Soni P, Li Y, et al (2017) Gene and transcript abundances of bacterial type III secretion systems from the rumen microbiome are correlated with methane yield in sheep. BMC Res Notes 10, ID 367.

38. Henderson G, Cox F, Ganesh S, et al (2015) Rumen microbial community composition varies with diet and host, but a core microbiome is found across a wide geographical range. Sci Rep 5, ID 14567. 